\title{
When Comorbidity, Aging, and Complexity of Primary Care Meet: Development and Validation of the Geriatric CompleXity of Care Index
}

\author{
Lillian Min, MD, MSHS, ${ }^{a, b}$ Neil Wenger, MD, ${ }^{c, d}$ Anne M. Walling, MD, PhD, ${ }^{c, d}$ Caroline Blaum, \\ MD, MS, ${ }^{e, f}$ Christine Cigolle, MD, MPH, ${ }^{a, b}$ David A. Ganz, MD, PhD, ${ }^{c, d, g, h}$ David Reuben, MD, ${ }^{g}$ \\ Paul Shekelle, MD, PhD, , d,h Carol Roth, RN, MPH, ${ }^{d}$ and Eve A. Kerr, MD, MPH ${ }^{i, j}$
}

OBJECTIVES: To develop and validate the Geriatric CompleXity of Care Index (GXI), a comorbidity index of medical, geriatric, and psychosocial conditions that addresses disease severity and intensity of ambulatory care for older adults with chronic conditions.

DESIGN: Development phase: variable selection and rating by clinician panel. Validation phase: medical record review and secondary data analysis.

SETTING: Assessing the Care of Vulnerable Elders-2 study.

PARTICIPANTS: Six hundred forty-four older ( $\geq 75)$ individuals receiving ambulatory care.

MEASURES: Development: 32 conditions categorized according to severity, resulting in 117 GXI variables. A panel of clinicians rated each GXI variable with respect to the added difficulty of providing primary care for an individual with that condition. Validation: Modified versions of previously validated comorbidity measures (simple count, Charlson, Medicare Hierarchical Condition Category), longitudinal clinical outcomes (functional decline, survival), intensity of ambulatory care (primary, specialty

From the a Division of Geriatrics, Department of Medicine, School of Medicine, University of Michigan, ${ }^{b}$ Geriatrics Research, Education and Clinical Care Center, Veterans Affairs Ann Arbor Healthcare System, Ann Arbor, Michigan; ${ }^{\mathrm{C}}$ Division of General Internal Medicine and Health Services Research, Department of Medicine, David Geffen School of Medicine, University of California at Los Angeles, Los Angeles, California; ${ }^{\mathrm{d}}$ RAND Health, Santa Monica, California; ${ }^{\mathrm{e}}$ Department of

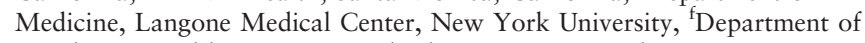
Population Health, Langone Medical Center, New York University, New York, New York; ' Division of Geriatrics, Department of Medicine, David Geffen School of Medicine, University of California at Los Angeles,

${ }^{\mathrm{h}}$ Greater Los Angeles Veterans Affairs Healthcare System, Los Angeles, California; ${ }^{i}$ Division of General Internal Medicine, Department of Medicine, School of Medicine, University of Michigan, and ${ }^{\mathrm{j} C e n t e r}$ for Clinical Management Research, Veterans Affairs Ann Arbor Healthcare System, Ann Arbor, Michigan.

Address correspondence to Dr. Lillian Min, Division of Geriatrics, Department of Medicine, University of Michigan, 300 North Ingalls Bldg Wing E, Room 966, Ann Arbor, MI 48109. E-mail: 1min@med.umich.edu

DOI: $10.1111 /$ jgs.12160 care visits, polypharmacy, number of eligible quality indicators (NQI)) over 1 year of care.

RESULTS: The most-morbid individuals (according to quintiles of GXI) had more visits (7.0 vs 3.7 primary care, 6.2 vs 2.4 specialist), polypharmacy $(14.3 \%$ vs $0 \%$ had $\geq 14$ medications), and greater NQI (33 vs 25 ) than the least-morbid individuals. Of the four comorbidity measures, the GXI was the strongest predictor of primary care visits, polypharmacy, and NQI $(P<.001$, controlling for age, sex, function-based vulnerability).

CONCLUSION: Older adults with complex care needs, as measured by the GXI, have healthcare needs above what previously employed comorbidity measures captured. Healthcare systems could use the GXI to identify the most complex elderly adults and appropriately reimburse primary providers caring for older adults with the most complex care needs for providing additional visits and coordination of care. J Am Geriatr Soc 61:542-550, 2013.

Key words: ambulatory care; utilization; comorbidity

$\mathrm{T}$ he medical home has been hailed as a new systembased strategy for providing high-quality patientcentered primary care, ${ }^{1}$ but one challenge that medical homes face will be to provide high-quality care to the older adults with the most complex care needs, typically those with multiple chronic conditions. ${ }^{2}$ Individuals with multimorbidity are at greater risk for mortality and disability than those with no major chronic conditions ${ }^{3,4}$ and generate greater healthcare costs. ${ }^{5,6}$ Beyond daily medication and self-care routines, individuals with multimorbidity have been described as having frequent pharmacy ${ }^{7}$ and doctor visits ${ }^{8,9}$ and multiple prescribers. ${ }^{7}$ Recent research has shifted attention to the potential burden of applying individual chronic disease guidelines to individuals with 
multiple chronic diseases. ${ }^{10,11}$ For clinicians, prioritizing patients' multiple conditions is a critical source of complexity. ${ }^{12}$ As health systems assume responsibility for delivering high-quality care to defined populations of individuals, understanding the intensity of care required to care for the older adults with the most-complex needs will become critically important.

The current approach to measuring multimorbidity and its effect on delivering high-quality care ignores geriatric conditions and the severity of individual diseases. ${ }^{13,14}$ Geriatric conditions are prevalent in older adults ${ }^{15}$ and are associated with functional decline $\mathrm{e}^{15}$ and poor quality of care. ${ }^{16}$ Therefore, as part of the Assessing the Care of Vulnerable Elders-2 (ACOVE- 2$)^{17}$ study, an effort was made to develop a new, more-comprehensive comorbidity index for older adults. The Geriatric CompleXity of Care Index (GXI) includes difficult-to-manage geriatric conditions (e.g., dementia and falls) and severity ratings for geriatric and other chronic diseases. This article describes the development and multistep validation of the GXI index (Figure 1).

ACOVE-2 was an ideal opportunity to study comorbidity and complexity of care. Participants had geriatric and other conditions and were evaluated for eligibility for a comprehensive set of ambulatory care quality indicators (QIs) as a measure of complexity of care. It was primarily hypothesized that the GXI would be a better predictor of complicated ambulatory care (number of eligible QIs and polypharmacy) than three previously employed comorbidity measures: a simple count of 12 conditions used in prior ACOVE studies, ${ }^{13,18,19}$ the Charlson Comorbidity Index (CCI) ${ }^{20}$ and Medicare Hierarchical Condition Categories (HCC). ${ }^{5}$ The secondary hypotheses were that the CCI would be the best at predicting clinical outcomes (function and survival) and that the HCC would be the best at predicting number of ambulatory care visits.

\section{METHODS}

\section{Development of the GXI}

First, candidate conditions were identified based on a nosology of diseases of aging. ${ }^{21}$ Candidate conditions were considered if they were chronic, symptomatic, and prevalent and required ambulatory care. Conditions were stratified according to disease severity, including subclinical forms (e.g., pre-diabetes mellitus (DM)), as less-severe versions of the conditions. When available, existing staging methods were used (e.g., New York Heart Association heart failure criteria2 ${ }^{22}$ ). In other cases, commonly documented symptoms were used as indicators of severity (e.g., behavior problems as a severity criterion for dementia). Combinations of common co-prevalent conditions in older adults were also considered as sources of additional complexity: hypertension and chronic kidney disease (CKD); ${ }^{23}$ $\mathrm{DM}$ and $\mathrm{CKD} ;{ }^{23}$ and depression, insomnia, and anxiety. ${ }^{24,25}$ In total, 146 candidate variables were considered

\section{DEVELOPMENT:}

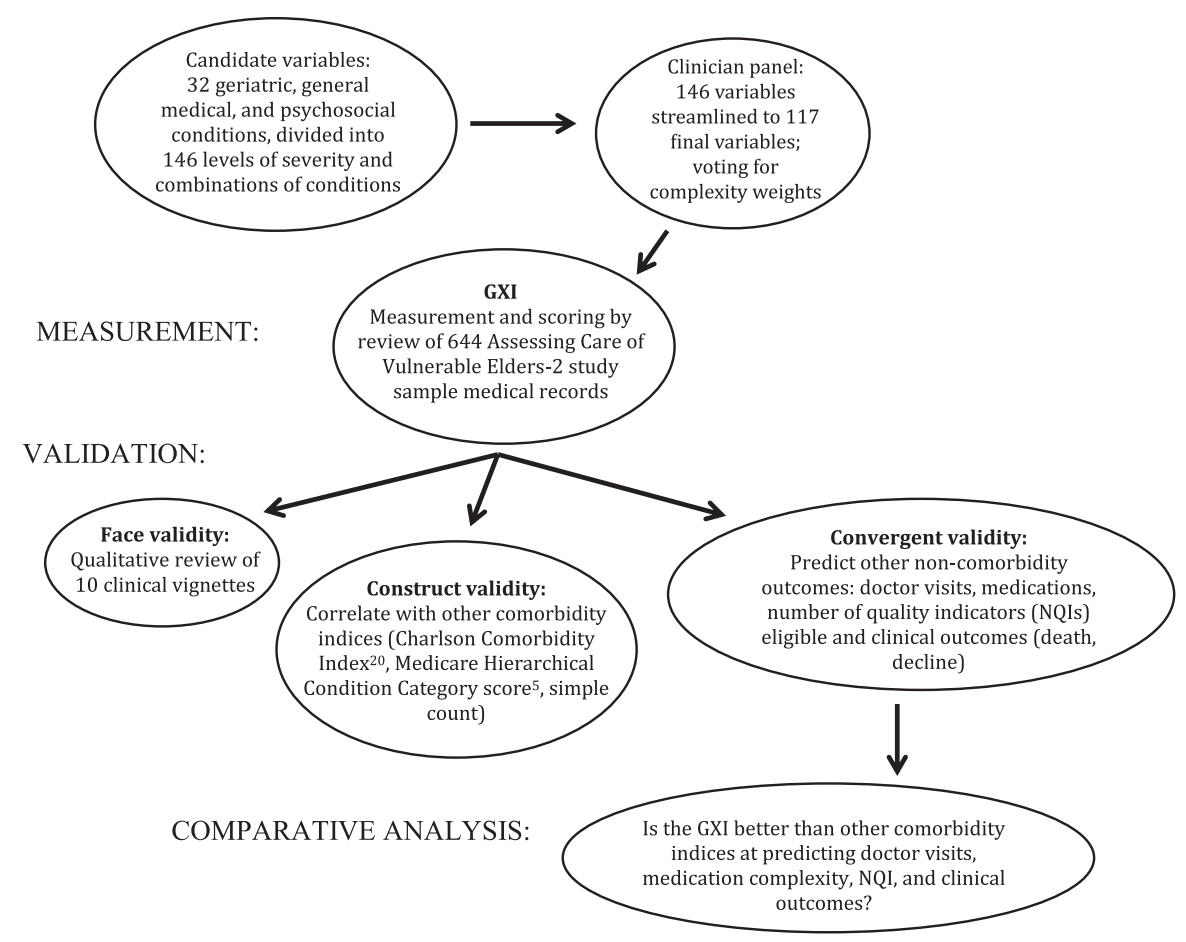

CCI: Charlson Comorbidity Index ${ }^{20}$

HCC: Medicare Hierarchical Condition Category score ${ }^{5}$

ACOVE-2: Assessing Care of Vulnerable Elders-2 study sample

Figure 1. Development and validation of the Geriatric CompleXity of Care Index (GXI). NQI = number of quality indicators. 
(32 conditions, stratified according to severity, with potential interactions between conditions and levels of severity).

\section{Expert panel assessment}

A panel of six clinician-researchers (including LM, DR, DG, PS, and NW) with experience in clinical care of older adults with complex care needs, quality measurement, and comorbidity was first convened. In two rounds of voting, the panelists first voted on inclusion of candidate variables and then weighted each condition according to their assessment of difficulty involved with caring for an older adult with that condition over 1 year of primary care. Each candidate variable was rated on a scale from 1 to 10, with higher scores representing a morecomplex form of the condition. Each GXI variable was assigned the median rating of the six raters as the final complexity weight. Two combinations of multiple conditions (DM and CKD; depression, anxiety, and insomnia) were rated as having more complexity than the sum of the individual conditions. The panel's final number of GXI variables (conditions, severity levels, and interactions) was 117 (Appendix 1).

\section{Setting and data collection}

Medical records from ACOVE-217, a practice-based intervention in 2002 that enrolled 644 individuals at two multisite ambulatory care practices in southern California, were reviewed. This cohort comprised older adults $(\geq 75)$ who had at least one of three geriatric conditions (urinary incontinence (UI), impaired memory, and falls). Within each practice, one site received a multicomponent practicedirected quality improvement intervention ${ }^{26}$ for dementia, falls, and UI; the other sites (one in the first practice, four at the other) served as controls (only patient screening).

In the original ACOVE-2 study, participants' complete ambulatory medical records (including all primary and specialty care providers for the 13-month study) were collected for evaluation of 98 ACOVE ambulatory care quality indicators (QIs) in 13 areas of medical and geriatric care (dementia, depression, DM, falls and fear of falling, hearing impairment, hypertension, malnutrition, osteoporosis, osteoarthritis, pain, UI, medication use, screening and prevention). ${ }^{27} \mathrm{~A}$ detailed medical record review was performed to determine eligibility for 65 QIs. In addition, 578 participants were interviewed upon enrollment, which determined eligibility for an additional 33 interview-based QIs.

For the current study, complete medical records were re-abstracted for the 117 GXI variables during the study year. Two abstractors (LM, AW) were blinded to which QIs were previously measured. ${ }^{16,17,27}$ The mean time needed to abstract a chart for the GXI was 12 minutes. The institutional review boards at RAND Health and the University of California at Los Angeles approved this study.

\section{Primary predictor variable: GXI comorbidity score}

Each individual's GXI score was calculated, based on his or her conditions, as the sum of the complexity weights as rated by the expert panel. A higher GXI indicated greater burden. For example, if an individual had uncomplicated atrial fibrillation $(\mathrm{AF})$ (complexity weight $=3$ ), uncomplicated emphysema (complexity weight $=4$ ), and hypercholesterolemia (complexity weight $=2$ ), the GXI score was calculated to be 9 .

\section{Outcome variables}

To validate the GXI, six outcome measures of clinical complexity were studied. These measures were not based on medical conditions and included two clinical outcomes, two provider visit variables, polypharmacy, and number of eligible QIs.

\section{Clinical outcomes}

Longitudinal functional decline and mortality outcomes had been previously collected on the ACOVE-2 cohort (2002-2007). ${ }^{28}$ Functional decline was defined using the Short Functional Survey (SES), ${ }^{29,30}$ which summarizes independence in five activities of daily living (shopping, light housework, finances, bathing, and walking; 0-5 possible points). Two 5-year outcomes were calculated for each individual: death versus survival and functional decline versus no decline. Full information was available on death outcomes. Information on functional decline outcomes was collected for 295 participants.

\section{Primary care provider and specialty care visits}

As a measure of ambulatory care use, available administrative data were used to identify the number of visits to the individual's primary care provider. If more visits were made to an alternate primary care provider, the alternate provider was assigned as the individual's primary care provider. Each individual's specialty visits over the same time period were counted.

\section{Number of eligible quality indicators}

Because each ACOVE ambulatory care QI specifies an individual's eligibility for an ambulatory care process, ${ }^{31}$ it was determined that the number of eligible QIs (NQI) for each individual could serve as a unique marker of the clinical care needed to meet quality standards.

The number of medical-record based ambulatory care QIs for which each individual was eligible (of 65 possible) was calculated for each individual. For participants who provided interview-based eligibility information, an alternative NQI was calculated that combined medical record and interview eligibility methods (98 QIs possible). As in the original ACOVE-2 study, individuals with advanced dementia and limited life expectancy were not eligible for a subset of QIs based on appropriateness criteria. ${ }^{32}$ Because nearly all QIs require more care (only 2 of 98 were "overuse"-type QIs), a higher NQI indicates an individual needing higher-intensity ambulatory care.

\section{Medication use}

As an additional measure of ambulatory care intensity, ${ }^{7}$ the maximum number of medications documented in the medical record at any visit was counted. Only prescribed medications or over-the-counter medications recommended on a longterm basis for chronic condition management were included. 
Polypharmacy was categorized as none ( $\leq 6$ medications), mild (6-8), moderate (9-12), or severe ( $\geq 14)$.

\section{Comparison comorbidity measures (simple count, CCI, and HCC)}

The ACOVE simple count consisted of 12 conditions: AF, coronary artery disease, heart failure, cerebrovascular disease, DM, hypertension, dementia, falls, hearing impairment, UI, malnutrition, and osteoporosis (starred in Appendix 1).

All 19 comorbidities from the $\mathrm{CCI}^{20}$ were mapped to GXI variables to calculate a modified CCI score (mCCI) for each individual. Claims data necessary to calculate the HCC directly (based on 80 acute and chronic conditions) were not available. To calculate a modified HCC score (mHCC), 37 ambulatory care conditions were mapped to the HCC, and previously published community-based weights were applied. Five for mCCI and mHCC, points were omitted for age and sex because these variables are not included in the GXI and simple count.

\section{Covariables}

Sex, age, ACOVE-2 intervention site, and the Vulnerable Elders Survey-13 (VES-13) were used in multivariable models. ${ }^{33}$ The VES-13 is a 13-item screening tool (11 items pertaining to physical abilities and activities of daily living, 1 item regarding self-rated health, and age) that predicts death and further functional decline. It contains no comorbidity variables and predicts outcomes independently of comorbidity. ${ }^{28,33,34}$

\section{Analysis}

To validate the GXI (Figure 1), first 10 comorbidity vignettes of ACOVE-2 participants were reviewed for face validity, then the GXI was correlated with previously validated comorbidity measures for construct validity (simple count, mHCC, and mCCI), and finally the GXI was analyzed against external (noncomorbidity) measures for convergent (predictive) validity and its performance was compared with the previously validated comorbidity measures.

\section{Qualitative review of 10 multimorbid individuals receiving ambulatory care}

To review for face validity, ACOVE-2 participants with increasing combinations of five conditions (hypertension, coronary artery disease, DM, AF, and dementia) were selected, a method based on prior comorbidity work. ${ }^{19}$ All individuals with none of the five conditions were identified as the first tier, those with hypertension and none of the remaining conditions as the second tier, and so on, up to individuals with all five conditions for the sixth tier. Two individuals were selected from each tier, one with a low (bottom tertile) GXI score, and one with a high (top tertile) GXI score, for qualitative review as comorbidity vignettes (Appendix 2).

\section{Quantitative analysis}

Correlations were first calculated between the GXI and the previously validated comorbidity measures. Next, to validate the GXI against the noncomorbidity measures, unadjusted effect (logistic and linear regression) was calculated on clinical outcomes, ambulatory care visits, and complexity of care measures for increasing quintiles of GXI scores (Table 2). Multivariable regression was used to introduce control variables (age, sex, VES-13, intervention site) and to calculate the effect of GXI (top minus bottom quintile, last columns of Table 2) on the external validation variables.

To compare how well the GXI predicted the external validation measures with the previously validated comorbidity variables, each comorbidity measure was paired with each external outcome using model fit (coefficient of determination; $R^{2}$ for linear regression models and area under the receiver operating characteristic curve (AUC) for logistic models) as the comparative metric (Table 3). Last, GXI and each previously validated comorbidity index was entered into fully adjusted models using tests of statistical independence $(P<.05)$ to evaluate whether the moreresource-intensive GXI score would better predict the outcomes than the previously validated comorbidity variables. All analyses were performed using Stata 12 (Stata Corp., College Station, TX).

\section{RESULTS}

Clinician panel ratings for 117 GXI comorbidity variables (Appendix 1; online version only)

The range of median ratings by the panel extended from 1 (history of smoking in a nonsmoker) to 8 (severe liver disease, new depression with symptoms of insomnia and anxiety, nonsevere dementia with behavior problems, and severe dementia with behavior problems).

\section{Qualitative review of 10 comorbidity vignettes} (Appendix 2; online version only)

Review of the vignettes confirmed that, within similar conditions, individuals selected for high complexity scores were substantively more complex in the nature of their conditions, medication regimens, and NQI and had more doctor visits.

As an example of how GXI distinguished clinical complexity, two individuals were identified as having hypertension but none of the four other conditions (coronary artery disease, DM, AF, and dementia; Tier 2, Appendix 2). Although one did not have complex care needs (2nd percentile on GXI score) and the other did (80th percentile), both had a simple condition count of 3 . The noncomplex individual had well-controlled hypertension ( 2 points added to GXI), normal weight (0 points), osteoporosis without fracture ( 2 points), hearing impairment ( 2 points), and falls (5 points). By contrast, the complex individual's hypertension was moderately severe (3 points), and she had chronic pain ( 5 points), hypothyroidism ( 2 points), hearing ( 2 points) and vision problems ( 3 points), fatigue ( 5 points), insomnia ( 2 points), and stable mild kidney disease ( 2 points). She developed a new osteoporotic fracture (6 points), weight loss (4 points), new anemia ( 2 points), and new UI (4 points). Despite the vast difference in complexity, the simple 


\section{Table 1. Sample Characteristics}

\begin{tabular}{|c|c|}
\hline Characteristic & Value \\
\hline \multicolumn{2}{|l|}{ Demographic } \\
\hline Age, mean \pm SD (range) & $81.2 \pm 4.8(75-100)$ \\
\hline Male, n (\%) & 217 (33.6) \\
\hline \multicolumn{2}{|l|}{ Clinical } \\
\hline $\begin{array}{l}\text { Vulnerable Elders Survey-13 score, } \\
\text { mean } \pm \text { SD (range) }{ }^{\mathrm{a}}\end{array}$ & $4.6 \pm 2.9(1-9)$ \\
\hline 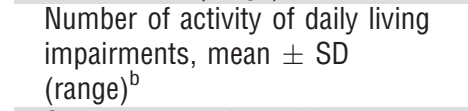 & $0.9 \pm 1.44(0-5)$ \\
\hline $\begin{array}{l}\text { Simple count of conditions, } \\
\text { mean } \pm S D{\text { (range })^{c}}^{c}\end{array}$ & $3.6 \pm 1.6(0-9)$ \\
\hline $\begin{array}{l}\text { Modified Charlson Comorbidity } \\
\text { Index, mean } \pm \text { SD (range) })^{d, 20}\end{array}$ & $3.7 \pm 2.6(0-11)$ \\
\hline $\begin{array}{l}\text { Modified Hierarchical Condition } \\
\text { Count score (predicted annual } \\
\text { cost), } \$ \text {, mean } \pm \text { SD (range) }\end{array}$ & $7,432 \pm 4,665(2,475-30,125)$ \\
\hline $\begin{array}{l}\text { Geriatric Complexity of Care score, } \\
\text { mean } \pm \text { SD (range) }\end{array}$ & $31.3 \pm 11.6(7-72)$ \\
\hline \multicolumn{2}{|l|}{ Health care } \\
\hline $\begin{array}{l}\text { Number of visits with assigned } \\
\text { primary care provider, } \\
\text { mean } \pm \text { SD (range) }\end{array}$ & $5.43 .3(0-20)$ \\
\hline $\begin{array}{l}\text { Number of visits with specialists, } \\
\text { mean } \pm \text { SD (range) }\end{array}$ & $3.95 .6(0-62)$ \\
\hline \multicolumn{2}{|c|}{ Polypharmacy (number of chronic medications), n (\%) } \\
\hline None $(\leq 6)$ & $316(49)$ \\
\hline Moderate (7-9) & $172(26.8)$ \\
\hline Severe $(10-13)$ & $117(18.2)$ \\
\hline Very severe $(\geq 14)$ & $39(6.1)$ \\
\hline $\begin{array}{l}\mathrm{NQI} \text { needed (as documented in } \\
\text { medical record), mean } \pm \mathrm{SD} \\
\text { (range) }\end{array}$ & $12.2 \pm 4.3(3-26)$ \\
\hline $\begin{array}{l}\mathrm{NQI} \text { (as documented in medical } \\
\text { record and in interviews), } \\
\text { mean } \pm \mathrm{SD} \text { (range) }{ }^{\mathrm{f}}\end{array}$ & $27.5 \pm 6.8(8-47)$ \\
\hline
\end{tabular}

The analytical sample is from the Assessing the Care of Vulnerable Elders2 Study, ${ }^{17}$ which originally screened 2,671 individuals aged $\geq 75$ for falls, urinary incontinence (UI), and memory impairment; 784 of these $(29 \%)$ screened positive for one or more of the geriatric conditions, and 649 $(83 \%)$ agreed to enroll in the study. Adequate medical records were obtained for $644(99 \%)$ to review for quality and complexity of care. The longitudinal outcomes of the overall sample have been previously reported: ${ }^{28} 220(34 \%)$ of the sample died. Two hundred ninety-five of the survivors were contacted for a functional status interview 5 years after enrollment.

a A function-based risk score predicting 1-, 2-, and 5-year risk of functional decline or death. ${ }^{28,33,34} \mathrm{~A}$ score of $\geq 3$ identifies individuals aged 65 or higher at the $30 \%$ highest risk.

${ }^{\mathrm{b}}$ Number of self-reported disabilities of 5 possible (shopping, light housework, walking, bathing, finances) reported as difficult and requiring help or not doing because of health according to the Short Functional Survey. ${ }^{29}$

${ }^{\mathrm{c}}$ Unweighted sum of atrial fibrillation, coronary artery disease, congestive heart failure, cerebrovascular disease, diabetes mellitus type 2, hypertension, dementia, falls, hearing impairment, UI, malnutrition, and osteoporosis.

d Charlson Comorbidity Index ${ }^{20}$ was calculated without age and sex points, using comorbidities only, analogous to the simple count and Geriatric CompleXity of Care Index score.

${ }^{\mathrm{e}}$ Comorbidity using dollar weights from the Medicare Hierarchical Condition Category ${ }^{5}$ score. The modified HCC did not include acute diseases, age, and sex weights.

${ }^{\mathrm{f}}$ The $\mathrm{n}$ for these variables was 578 who participated in the quality-of-care interview.

$\mathrm{SD}=$ standard deviation; $\mathrm{NQI}=$ number of quality indicators condition count did not indicate a difference in complexity between these two individuals.

\section{Quantitative results}

Sample characteristics are described in Table 1.

The GXI was correlated with all of the previously validated comorbidity scores $(P<.001)$ : simple count $(r=.66)$, mCCI $(r=.62)$, and $\mathrm{mHCC}(r=.73)$.

The GXI defined increasing care intensity, with substantial and statistically significant tests of trends across all of the unadjusted intensity variables $(P<.001$ for all except functional decline, $P<.02$ ) (Table 2 ). An individual in the top quintile of GXI, on average, visited her primary care provider 7.0 times, versus 3.7 times for an individual in the bottom quintile. No individuals in the healthiest quintile had the most-severe category of polypharmacy, but $14 \%$ of those in the highest quintile were in this category. The mean NQI was 16 for the most complex quintile and 9 for the least complex. Although the GXI was associated with functional decline and survival on bivariate analysis, it was not as strong as the VES-13 in the multivariable model (last column, Table 2) and became nonsignificant in adjusted models.

When comparing the GXI with the other comorbidity variables at predicting outcomes (using model fit as the criterion), the GXI was comparable with or worse than previously validated indices for predicting death and decline but better at predicting care intensity: doctor visits, polypharmacy, and NQI (defined as an $R^{2}$ or AUC of $>5 \%$ points better than at least one other index, Table 3), with polypharmacy reaching the excellent AUC range $(>0.8)$. The GXI also independently $(P<.01)$ predicted primary care and specialty visits, polypharmacy, and NQI, controlling for age, sex, intervention site, and VES-13 and when any of the three previously validated comorbidity measures (simple count, mCCI, mHCC) was also entered into the model (not shown). The mHCC was the best predictor $(P<.001$, even with any other three comorbidity variables included) of death. None of the comorbidity variables predicted 5-year functional decline independent of VES-13 (odds ratio $=2.0$ per point, $P<.001$ for all models).

\section{DISCUSSION}

This article describes the multistage development of a comprehensive measure of geriatric comorbidity, the GXI. The GXI predicted ambulatory care intensity, as measured according to primary and specialty care visits, polypharmacy, and eligibility for complex ambulatory care processes independent of age, functional status, and previously validated measures of comorbidity. The GXI captured complex care as measured according to NQI and complexity of medication management well. The authors believe that they were successful at capturing ambulatory care intensity with the GXI because geriatric conditions and severity of disease were considered.

In contrast with other comorbidity studies, ${ }^{5,6,12,20}$ the GXI was also tested against functional status, an important outcome for older adults. Functional impairment has also been included as a condition in other comorbidity indices. $^{35,36}$ Although comorbidity and function are 
Table 2. Mean Clinical and Intensity-of-Care Variables Across Quintiles of Geriatric CompleXity of Care Index (GXI) in Assessing Care of Vulnerable Elders 2

\begin{tabular}{|c|c|c|c|c|c|c|c|}
\hline Variable & \multicolumn{6}{|c|}{$\begin{array}{l}\text { Mean (Unadjusted) Values Across Quintiles of GXI (Range of GXI Points } \\
\text { for Individuals in Quintile) } n\end{array}$} & $\begin{array}{c}\text { Adjusted Difference } \\
\text { Between Top and } \\
\text { Bottom Quintiles } \\
\text { Effect Size }{ }^{\mathrm{a}}(95 \% \\
\text { Confidence Interval) }\end{array}$ \\
\hline 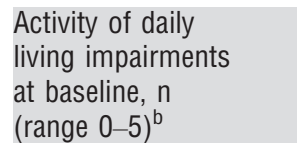 & 0.3 & 0.7 & 1.2 & 1.1 & 1.3 & $<.001$ & 0.8 (0.5 to 1.1$)$ \\
\hline $\begin{array}{l}\text { VES-13 score at } \\
\text { baseline (range } 0-10 \text {, } \\
\text { higher }=\text { greater risk) }\end{array}$ & 3.1 & 3.9 & 5.1 & 5.2 & 5.6 & $<.001$ & 2.0 (1.6 to 2.5$)$ \\
\hline Died (5-year), \% & 21.5 & 23.2 & 36.4 & 42.5 & 45.1 & $<.001$ & $4.8(0$ to 14$)$ \\
\hline $\begin{array}{l}\text { Functional decline } \\
\text { (5-year), } \%^{\mathrm{d}}\end{array}$ & 4.5 & 4.1 & 11.1 & 11.5 & 14.3 & .02 & $2.5(-2.4$ to 3.1$)$ \\
\hline $\begin{array}{l}\text { Medical record } \\
\text { Qls eligible per } \\
\text { individual, } \mathrm{n}\end{array}$ & 9.2 & 10.4 & 12.0 & 13.0 & 15.9 & $<.001$ & $7.1(6.1$ to 8.4$)$ \\
\hline $\begin{array}{l}\text { All QIs eligible from } \\
\text { medical record and } \\
\text { interview, } n(n=578)^{f}\end{array}$ & 23.5 & 26.0 & 27.4 & 28.5 & 31.9 & $<.001$ & 9.7 (8.0 to 11.3$)$ \\
\hline
\end{tabular}

${ }^{a}$ Predicted outcomes for top versus bottom quintile of GXI, controlling for age, sex, Vulnerable Elders Survey-13 (VES-13), and intervention group. GXI was a five-category variable. Confidence intervals were obtained using bootstrapping. VES-13 was omitted as a control variable to predict VES-13 and activity of daily living disabilities because of colinearity.

${ }^{\mathrm{b}}$ Functional status as measured according to the Short Functional Survey (SFS). ${ }^{29}$

${ }^{\mathrm{c}}$ A scale predicting risk of $1-^{34}, 2{ }^{33}$, and $5^{34}$-year risk of functional decline and death.

${ }^{\mathrm{d}}$ Five-year functional decline was defined as SFS decline of $\geq 1$ abilities. ${ }^{29,30}$ Two hundred ninety-five survivors participated in the follow-up SFS. The reduced quintile sample sizes for the 5 -year functional decline outcome was (1st through 5 th): 66, 74, 54, 52, 59.

${ }^{\mathrm{e}}$ Includes routine prescriptions for chronic conditions, "as needed" if taken on more than half of days, and nutritional supplements for management of chronic conditions.

${ }^{\mathrm{f}}$ Based on a sample size of 578, the number interviewed. The reduced quintile sample sizes were (1 through 5): 99, 124, 120, $120,115$. $\mathrm{QI}=$ quality improvement.

Table 3. Comparison Between the Geriatric CompleXity of Care Index (GXI) and Previously Validated Comorbidity Indexes in Predicting Clinical and Healthcare Outcomes

\begin{tabular}{|c|c|c|c|c|c|c|c|c|}
\hline & Simple Count & $\mathrm{mCCl}$ & $\mathrm{mHCC}$ & GXI & Simple Count & $\mathrm{mCCl}$ & $\mathrm{mHCC}$ & GXI \\
\hline Outcome & \multicolumn{4}{|c|}{ Unadjusted (\%) } & \multicolumn{4}{|c|}{ Adjusted (\%) } \\
\hline Primary clinician visits & 6.1 & 3.6 & 5.9 & $13.6^{\mathrm{a}}$ & 6.4 & 3.8 & 6.1 & $14.3^{\mathrm{a}}$ \\
\hline Specialty visits & 2.5 & 2.0 & 9.1 & 8.2 & 4.0 & 2.9 & 9.9 & $9.1^{\mathrm{a}}$ \\
\hline NQI (medical record only) & 33.2 & 17.7 & 14.3 & $30.5^{\mathrm{a}}$ & 35.2 & 19.6 & 15.8 & $32.8^{\mathrm{a}}$ \\
\hline 5-year mortality & 64.7 & 67.9 & 69.1 & 61.8 & 77.1 & 77.5 & 78.7 & 76.2 \\
\hline 5-year functional decline & 51.7 & 61.4 & 56.8 & 56.3 & 89.6 & 89.9 & 89.6 & 83.8 \\
\hline Polypharmacy ( $\geq 14$ medications) & 72.2 & 59.7 & 73.9 & $78.0^{\mathrm{a}}$ & 76.2 & 70.1 & 76.9 & $81.5^{\mathrm{a}}$ \\
\hline
\end{tabular}

Adjusted models controlled for age, sex, vulnerability, and intervention versus control site.

${ }^{a}$ GXI $R^{2}$ or area under the receiver operating characteristic curve (AUC) at least 5 percentage points better than any one of the previously validated comorbidity indices.

$\mathrm{mCCI}=$ modified Charlson Comorbidity Index,${ }^{20}$ risk of death calculated without age; GXI = Geriatric CompleXity of Care Index; $\mathrm{mHCC}=$ modified Medicare-Hierarchical Condition Category, ${ }^{5}$ predicted annual cost for community-based sample, calculated without acute diseases, age, and sex; $\mathrm{NQI}=$ number of quality indicators. 
important health concerns for older adults, by keeping them separate, it was possible to evaluate their relative contributions to the various outcomes tested. As an example, the GXI was independent of the VES-13, the function-based covariable, at predicting NQI, but GXI was less predictive than VES-13 at predicting future decline. This is consistent with prior work, in which it was found that the VES-13 was an effective and parsimonious way to predict clinical outcomes in older community-dwelling adults and that comorbidity added no predictive value. ${ }^{33,34}$

Unlike other comorbidity measures that give more weight to acute medical and surgical conditions to predict death $^{20}$ and cost, ${ }^{5,6}$ the current study gave greater weight to the more difficult-to-manage geriatric conditions from the perspective of primary care clinicians. Therefore, the GXI complements the other indices by focusing on a different, but important, area of care-ambulatory care of older adults with multiple chronic conditions. In this study, the GXI was the best predictor of number of visits a primary care clinician will need to provide in a given year based on the complexity of his or her patients or a way to target valuable medical home resources for individuals with more-complex needs, such as pharmacy review or added care coordination.

The GXI was not as good as the other indices at predicting survival or functional decline, underscoring prior findings that no comorbidity index can predict all outcomes for all individuals. ${ }^{37}$ The mHCC, originally derived ${ }^{5}$ to adjust for high-cost individuals, was the best predictor of survival in this sample, even though not all of the acute illness variables necessary to compute a full score were available. It is not surprising that cost and mortality would be closely related, given accelerated costs at the end of life. $^{38,39}$ The results suggest that the mHCC would be a more-efficient way to risk-adjust for death outcomes than the GXI. Additionally, the mHCC outperformed the GXI at predicting specialty visits, consistent with the known relationship between mHCC and higher-cost care.

This study highlights the value of NQI, which quantifies the number of care processes that should be provided for an individual as an outcome variable. In prior studies, NQI was used as a proxy variable for comorbidity (as a predictor) rather than as an outcome. ${ }^{40}$ This analysis validates that it is reasonable to use NQI as a proxy for disease burden.

There are several limitations to this study. First, because this study of comorbidity was planned as a medical record abstraction, the GXI variable definitions were customized to what is typically documented in handwritten or dictated primary care records. Although the GXI was not difficult to collect (12 minutes on average per record), future refinement should occur within evolving health systems that can routinely capture required data. This study provides new insight into which comorbidities should be candidates for systemwide automated data to identify individuals requiring the most complex care. This would also facilitate further validation with other measures of complexity important to healthcare systems such as hospitalizations.

Second, the International Classification of Diseases (ICD) codes used to score the HCC were not available, so mapping of HCC variables to the chart-abstracted information was relied on. A future study is needed to develop an ICD version of the GXI for comparison with the HCC, although ICD codes should not be the criterion standard for measuring geriatric comorbidity because ICD codes do not capture important geriatric conditions such as falls.

A third potential limitation is that the ACOVE-2 study enrolled individuals with at least one geriatric condition, so the results of the current study might not be generalizable to unselected older populations. Inclusion of individuals with geriatric conditions increased the GXI for all participants in the sample, therefore if an unselected population with greater variation in GXI were re-enrolled, it would be expected that association with greater difference in intensity of primary care would be found. Fourth, the data were collected from 2002 to 2003, and practice patterns may have evolved (e.g., with respect to number of visits). Fifth, the sample was recruited from only two communities in southern California, and participants were nearly all white and had at least a high school education. The influence of ethnic and socioeconomic diversity on the predictive ability of this comorbidity index cannot be captured in this study. Sixth, this study focused on primary care rather than all health care. Unlike the original ACOVE-1 cohort, ${ }^{16}$ ACOVE-2 did not include the ACOVE hospital and continuity of care QIs that contribute greatly to the overall complexity of medical care managed by a medical home or healthcare system. How the GXI relates to acute care measures of complexity (e.g., transitions and readmissions) requires further study. Last, the number of potential ACOVE QIs doubled in 2007, ${ }^{41}$ mostly because of inclusion of new conditions; therefore, NQI will increase even more for individuals with complex needs as a result of the newer QI set.

In conclusion, detailed comorbidity and severity-ofdisease measures were used to capture the breadth of complex ambulatory care due to geriatric, psychosocial, and general medical conditions. The GXI can identify older adults with complex care needs who require adequate time and resources to provide them the ambulatory care that they need. With refinement for use in an electronic health record, the GXI could prospectively identify individuals who need more attention from future medical homes.

\section{ACKNOWLEDGMENTS}

We would like to acknowledge the technical assistance of Patty Smith and Caren Kamberg during the medical record review process. The clinician panel included authors Lillian Min, David Reuben, David Ganz, Paul Shekelle, Neil Wenger, and Joel Belmin from the Geriatric Service, Hôpital Charles-Foix, Assistance Publique Hôpitaux de Paris, Ivry sur Seine, France.

Conflict of Interest: This project was supported by the Agency for Healthcare Research and Quality (Min R21 HS017621). Dr. Min was supported by the Claude Pepper Older Americans Independence Centers at the University of California at Los Angeles (NIA-K12 2006-2010) and the University of Michigan (2010-2011) and a HartfordAFAR Geriatric Scholars Research Outcomes Award (2005-2006). Dr. Ganz is funded by a Veterans Affairs Greater Los Angeles Health Services Research and Development Center of Excellence Career Development Award 
(CD2 08-012-1). The original ACOVE-2 study was supported by a contract from Pfizer, Inc. to RAND. These results were presented at the American Geriatrics Society and Society for General Internal Medicine Meetings in 2012.

Author Contributions: Lillian Min and Anne Walling: study concept and design, acquisition of data, analysis and interpretation of data, and preparation of manuscript. Neil Wenger: study concept and design, acquisition of subjects and data, analysis and interpretation of data, and preparation of manuscript. Caroline Blaum: study concept and design, interpretation of data and preparation of manuscript. Chris Cigolle: interpretation of data and preparation of manuscript. David Ganz: study concept and design, acquisition of data, interpretation of data, and preparation of manuscript. David Reuben and Paul Shekelle: study concept and design, acquisition of subjects and data, interpretation of data, and preparation of manuscript. Carol Roth: acquisition of subjects and data, interpretation of data, and preparation of manuscript. Eve Kerr: study concept and design, analysis and interpretation of data, and preparation of manuscript.

Sponsor's Role: Neither RAND nor Pfizer participates in the design, analysis, or preparation of this manuscript.

\section{REFERENCES}

1. Stange KC, Nutting PA, Miller WL et al. Defining and measuring the patient-centered medical home. J Gen Intern Med 2010;25:601-612.

2. Rich ELD, Libersky J, Parchman M. Coordinating Care for Adults With Complex Care Needs in the Patient-Centered Medical Home: Challenges and Solutions. White Paper (Prepared by Mathematica Policy Research under Contract No HHSA290200900019I/HHSA29032005T) AHRQ Publication No 12-0010-EF. Rockville, MD: Agency for Healthcare Research and Quality, 2012.

3. Gijsen R, Hoeymans N, Schellevis FG et al. Causes and consequences of comorbidity: A review. J Clin Epidemiol 2001;54:661-674.

4. de Groot V, Beckerman H, Lankhorst GJ et al. How to measure comorbidity. A critical review of available methods. J Clin Epidemiol 2003;56: 221-229.

5. Pope GC, Kautter J, Ellis RP et al. Risk adjustment of Medicare capitation payments using the CMS-HCC model. Health Care Financ Rev 2004;25:119-141.

6. Weiner JP, Starfield BH, Lieberman RN. Johns Hopkins Ambulatory Care Groups (ACGs). A case-mix system for UR, QA and capitation adjustment. HMO Pract 1992;6:13-19.

7. Choudhry NK, Fischer MA, Avorn J et al. The implications of therapeutic complexity on adherence to cardiovascular medications. Arch Intern Med 2011;171:814-822.

8. Starfield B, Lemke KW, Herbert R et al. Comorbidity and the use of primary care and specialist care in the elderly. Ann Fam Med 2005;3:215222.

9. Starfield B, Lemke KW, Bernhardt T et al. Comorbidity: Implications for the importance of primary care in 'case' management. Ann Fam Med 2003;1:8-14.

10. Boyd CM, Darer J, Boult C et al. Clinical practice guidelines and quality of care for older patients with multiple comorbid diseases: Implications for pay for performance. JAMA 2005;294:716-724.

11. Tinetti ME, Bogardus ST Jr, Agostini JV. Potential pitfalls of diseasespecific guidelines for patients with multiple conditions. N Engl J Med 2004;351:2870-2874.

12. Grant RW, Ashburner JM, Hong CC et al. Defining patient complexity from the primary care physician's perspective: A cohort study. Ann Intern Med 2011;155:797-804.

13. Higashi T, Wenger NS, Adams JL et al. Relationship between number of medical conditions and quality of care. N Engl J Med 2007;356: 2496-2504.

14. Fung CH, Setodji CM, Kung FY et al. The relationship between multimorbidity and patients' ratings of communication. J Gen Intern Med 2008;23:788-793.
15. Cigolle CT, Langa KM, Kabeto MU et al. Geriatric conditions and disability: The Health and Retirement Study. Ann Intern Med 2007;147:156-164.

16. Wenger NS, Solomon DH, Roth CP et al. The quality of medical care provided to vulnerable community-dwelling older patients. Ann Intern Med 2003;139:740-747.

17. Wenger NS, Roth CP, Shekelle PG et al. A practice-based intervention to improve primary care for falls, urinary incontinence, and dementia. J Am Geriatr Soc 2009;57:547-555.

18. Min LC, Reuben DB, MacLean $\mathrm{CH}$ et al. Predictors of overall quality of care provided to vulnerable older people. J Am Geriatr Soc 2005;53:17051711.

19. Min LC, Wenger NS, Fung C et al. Multimorbidity is associated with better quality of care among vulnerable elders. Med Care 2007;45:480-488.

20. Charlson ME, Pompei P, Ales KL et al. A new method of classifying prognostic comorbidity in longitudinal studies: Development and validation. J Chronic Dis 1987;40:373-383.

21. Karlamangla A, Tinetti M, Guralnik J et al. Comorbidity in older adults: Nosology of impairment, diseases, and conditions. J Gerontol A Biol Sci Med Sci 2007;62A:296-300.

22. Hunt SA, Baker DW, Chin MH et al. ACC/AHA guidelines for the evaluation and management of chronic heart failure in the adult: Executive summary. A report of the American College of Cardiology/American Heart Association Task Force on Practice Guidelines (Committee to revise the 1995 Guidelines for the Evaluation and Management of Heart Failure). J Am Coll Cardiol 2001;38:2101-2113.

23. Kalaitzidis R, Li S, Wang C et al. Hypertension in early-stage kidney disease: An update from the Kidney Early Evaluation Program (KEEP). Am J Kidney Dis 2009;53:S22-S31.

24. Taylor DJ, Lichstein KL, Durrence HH et al. Epidemiology of insomnia, depression, and anxiety. Sleep 2005;28:1457-1464.

25. Neckelmann D, Mykletun A, Dahl AA. Chronic insomnia as a risk factor for developing anxiety and depression. Sleep 2007;30:873-880.

26. Reuben DB, Roth C, Kamberg C et al. Restructuring primary care practices to manage geriatric syndromes: The ACOVE-2 intervention. J Am Geriatr Soc 2003;51:1787-1793.

27. Ganz DA, Wenger NS, Roth CP et al. The effect of a quality improvement initiative on the quality of other aspects of health care: The law of unintended consequences? Med Care 2007;45:8-18.

28. Min L, Yoon W, Mariano J et al. The Vulnerable Elders-13 Survey predicts 5 -year functional decline and mortality outcomes in older ambulatory care patients. J Am Geriatr Soc 2009;57:2070-2076.

29. Saliba D, Orlando M, Wenger NS et al. Identifying a short functional disability screen for older persons. J Gerontol A Biol Sci Med Sci 2000;55A: M750-M756.

30. Min LC, Wenger NS, Reuben DB et al. A short functional survey is responsive to changes in functional status in vulnerable older people. J Am Geriatr Soc 2008;56:1932-1936.

31. Shekelle PG, MacLean CH, Morton SC et al. Assessing care of vulnerable elders: Methods for developing quality indicators. Ann Intern Med 2001;135:647-652.

32. Solomon DH, Wenger NS, Saliba D et al. Appropriateness of quality indicators for older patients with advanced dementia and poor prognosis. J Am Geriatr Soc 2003;51:902-907.

33. Saliba D, Elliott M, Rubenstein LZ et al. The Vulnerable Elders Survey: A tool for identifying vulnerable older people in the community. J Am Geriatr Soc 2001;49:1691-1699.

34. Min LC, Elliott MN, Wenger NS et al. Higher Vulnerable Elders Survey scores predict death and functional decline in vulnerable older people. J Am Geriatr Soc 2006;54:507-511.

35. Rockwood K, Song X, MacKnight C et al. A global clinical measure of fitness and frailty in elderly people. Can Med Assoc J 2005;173:489-495.

36. Song X, Mitnitski A, MacKnight $\mathrm{C}$ et al. Assessment of individual risk of death using self-report data: An artificial neural network compared with a frailty index. J Am Geriatr Soc 2004;52:1180-1184.

37. Byles JE, D'Este C, Parkinson L et al. Single index of multimorbidity did not predict multiple outcomes. J Clin Epidemiol 2005;58:997-1005.

38. Riley GF, Lubitz JD. Long-term trends in Medicare payments in the last year of life. Health Serv Res 2010;45:565-576.

39. Hoover DR, Crystal S, Kumar R et al. Medical expenditures during the last year of life: Findings from the 1992-1996 Medicare current beneficiary survey. Health Serv Res 2002;37:1625-1642.

40. Min LC, Reuben DB, Keeler E et al. Is patient-perceived severity of a geriatric condition related to better quality of care? Med Care 2011;49: 101-107.

41. Wenger NS, Roth CP, Shekelle P. Introduction to the Assessing Care of Vulnerable Elders-3 quality indicator measurement set. J Am Geriatr Soc 2007;55(Suppl 2):S247-S252. 


\section{SUPPORTING INFORMATION}

Additional Supporting Information may be found in the online version of this article:

Appendix S1. Conditions, severity levels, prevalence, and complexity of care weights used in the geriatric complexity of care (GXI) score.
Appendix S2. A case series of comorbidity and complexity in assessing care of vulnerable elders 2 .

Please note: Wiley-Blackwell is not responsible for the content, accuracy, errors, or functionality of any supporting materials supplied by the authors. Any queries (other than missing material) should be directed to the corresponding author for the article 